UCRL-CR-128808

S/C-B336593

\title{
Design of a Very Low Inventory Tritium Fill System for NIF Cryogenic Targets
}

\section{Material Support Agreement B336593}

\section{Stage 1 Final Report}

\author{
M. A. Strzhemechny
}

Verkin Institute for Low Temperature Physics \&

Engineering Academy of Sciences of Ukraine

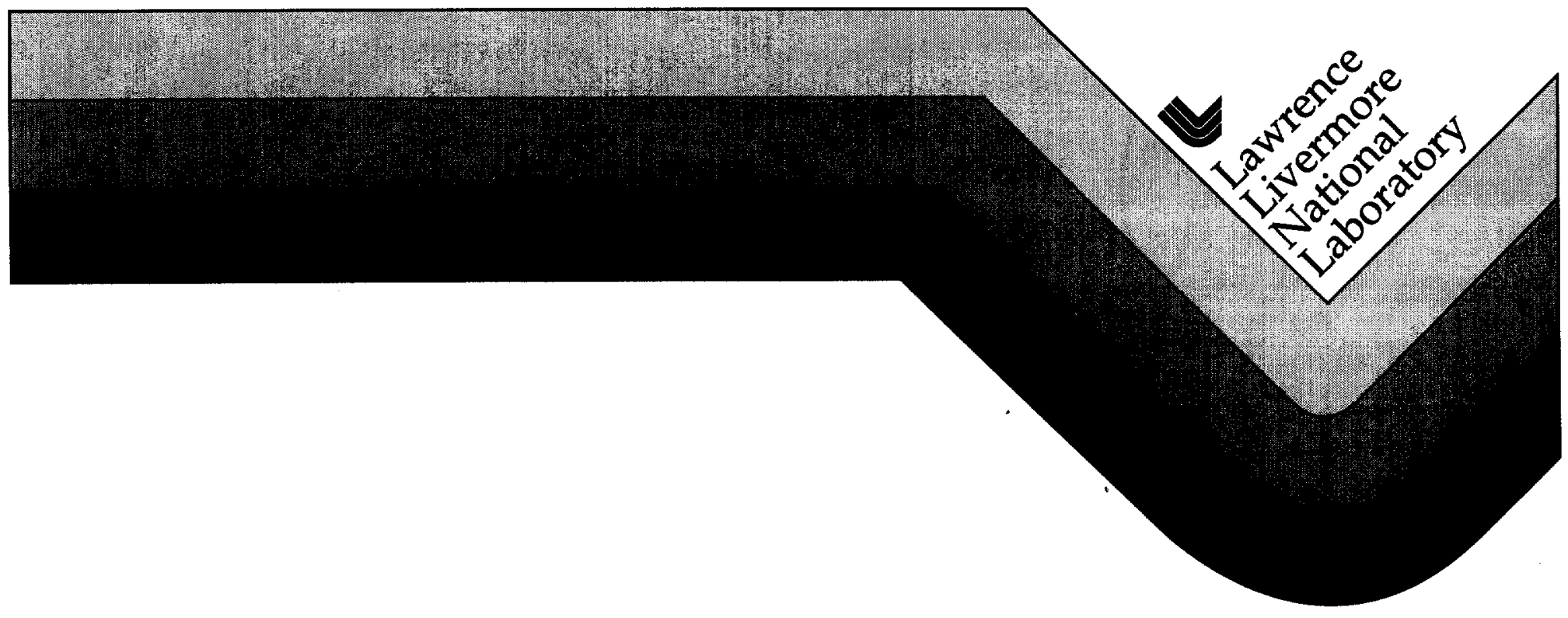




\section{DISCLAIMER}

This document was prepared as an account of work sponsored by an agency of the United States Government. Neither the United States Government nor the University of California nor any of their employees, makes any warranty, express or implied, or assumes any legal liability or responsibility for the accuracy, completeness, or usefulness of any information, apparatus, product, or process disclosed, or represents that its use would not infringe privately owned rights. Reference herein to any specific commercial product, process, or service by trade name, trademark, manufacturer, or otherwise, does not necessarily constitute or imply its endorsement, recommendation, or favoring by the United States Government or the University of California. The views and opinions of authors expressed herein do not necessarily state or reflect those of the United States Government or the University of California, and shall not be used for advertising or product endorsement purposes.

Work performed under the auspices of the U.S. Department of Energy by Lawrence Livermore National Laboratory under Contract W-7405-ENG-48. 


\title{
Material Support Agreement B 336593
}

\section{Stage 1 Final Report}

\author{
Date Angust 19, 1997 (Date due, August 17, 1997) \\ Signed by Psemedny (Dr. M. A. Strzhemechny)
}




\section{Agreement B336593}

\section{Stage 1 Final Report}

Design of a Very Low Inventory Tritium Fill System for NIF Cryogenic Targets

\section{Introduction}

In this report we finalize our conclusions concerning the issues that determine the feasibility of a low-inventory NIF target filling system:

1) calculation of the basic physical (thermodynamic, thermal flow, strength, etc.) characteristics;

2) choice of the optimum technological sequence;

3) choice of the principal design.

To make discussion easier, in this report we present more or less detailed sketches of the filling system assembly in explicit form. Since some of the already available important physical-engineering solutions (for instance, the design of the capsule removal device) are not know to us, we left certain engineering questions open for further discussion and detalization.

Summing up very briefly, we show that a very low-inventory filling system is undoubtedly feasible, notwithstanding some lingering ambiguities (for example, choice of the auxiliary capacity design, pressure gauge principle, etc.).

Our previous evaluation [1] of the general concept of the low-inventory filling system was based on calculations for normal $D_{2}$. The recalculation to the $50-50 \% D_{2}-T_{2}$ mixture, reported here, does not change much in either final figures, or conclusions.

We do not formulate anew the overall concept but refer to Part III of Agreement. We either omit some of our previous reasonings and evaluations, referring the reader to 1 st Quarterly Report [1].

\section{Basic Parameters and Specifications}

Capsule (hollow sphere)

Outer radius, $r$,

Wall thickness, $h$,

Total volume, $v$,
$0.1 \mathrm{~cm}$

$$
\begin{aligned}
& 10^{-2}-10^{-3} \mathrm{~cm} \\
& 4.19 \times 10^{-3} \mathrm{cc}
\end{aligned}
$$


Material

typical plastic

Ultimate pressure for buckling instability, $P_{e}$, $1-5$ bar

Tensile strength, $P_{t}$, $600-800$ bar

Yield stress, $\sigma_{f}$,

90-800 bar

Working body

Fluid (gas and liquid) $50 \%-50 \% \mathrm{D}_{2}-\mathrm{T}_{2}$ mixture

Mean density in filled capsule, $\bar{\rho}, 1.36 \times 10^{-2} \mathrm{moles} / \mathrm{cc}$

Chamber (cylindrical shape)

Inner radius, $r_{c}, \quad \quad 0.4 \mathrm{~cm}$

Height, $h_{c}$,

$0.4 \mathrm{~cm}$

Total volume, $v_{c}, \quad 0.201 \mathrm{cc}$

Volume of chamber space outside capsule, $V_{c}, \quad 0.197 \mathrm{cc}$

\section{Basic Engineering Design}

Making use of the results presented in our previous report [1], the objective of which was to calculate the relevant physical characteristics involved in the engineering design of the low-inventory filling system, we suggest the following general layout of the whole assembly (see Figs. 1 to 3 ) for two variants of the auxiliary capacity design, viz., the cylinder-piston (Fig. 1) or fixed-volume (Fig. 2) ones. Figure 3 is a blowup of the chamber unit, which houses the capsule during filling. We do not show some minor devices (some valves, for one) in order to avoid unnecessary overcrowding. We focus on some of the major issues that need choice below, under separate subdivision headings.

The cylinder-piston (or syringe) design (Fig. 1) features the corresponding unit comprising a cylinder (1 is the cylinder wall) and piston (2), intended to vary the volume of the empty space (3) employed to maintain the pressure within the fill chamber (4) (see also Fig. 3) at a desired level. The chamber cover (5) is depicted conditionally, since we are ignorant of the details and even operating principles of the existing capsule removal device or mechanism (we show only the flange that could be disconnected to provide access to both units). For the same reason the upper part of the housing is shown broken. Both the chamber and piston units are operating in vacuum in oder to minimize the unwanted uncontrollable heat inflows or outflows. To the same end serve the thermal isolation columns (6), which rest on a sufficiently powerful support slab or plate (9) (see also Subsec. 3.1). The heat path between both units and the cold bus (kept at approximately $20 \mathrm{~K}$ ) of sufficient heat-absorbing capacity are the high-thermal-conductivity metal rods or bands (7), as convenient. We show heat switches (8) as mechanical devices but the choice of their operating principle is optional. 
The chamber and the space under the piston communicate via the duct (10). Near the point where the duct enters the fill chamber, it meets another communication line duct (11), which leads to a pressure gauge (12) (see also Subsec. 3.2).

Our design features additional cooling facilities, which are independent helium flow cryostats (13); the corresponding helium transport lines (14) are also shown in the sketches. These facilities are necessary (see also Subsec. 3.3) when the temperature of both units needs to be varied fast enough.

The design of the version that incorporates a fixed-volume auxiliary capacity (Fig. 2) is basically the same as that of the piston version except for the absence of the powerful thermal-isolating columns, which are now not necessary because there is no need for a substantial force to be exerted upon the unit.

Item 15 shown in Fig. 3 is a pocket for the thermometer. Similar pockets can be found in Figs. 1 and 2 in other places.

3.1. Assembly-Carrying Support. The design depicted for both variants implies that the mechanism employed to remove filled capsules will need some device to exert stopping force upon the cover in order to withstand the pressure inside the chamber (on the order of half a kilobar) and, generally, to separate the chamber space from the inside of the housing. Of course, there can be other engineering solutions that would not necessitate application of an unpaired force, which entails inevitably a support. Instead, a pair of counteracting forces can be produced in other ways. This point depends strongly on the design of the capsule removal assembly.

3.2. Chamber Pressure Pickup. The version of the pressure measuring device as shown in Figs. 1 and 2 is the simplest solution of the problem. This problem is unavoidable because the seemingly only way to control the capsule filling process is by continuously monitoring the pressure inside the chamber (or in any other place, for by design the pressure is the same everywhere within the working body). The clear idea behind what is shown in the drawing is to create a separate space in which pressure measurements will be taken but at the expense of increasing noticeably (by $20 \%$ to $50 \%$ ) the amount of the D-T mixture used in one cycle. This increase leaves us within the allowable limits (see below). In this case the pressure can be measured in any appropriate way (membrane capacitance, using pressure dependence of the resistance of a long enough special wire, or employing a number of standard calibrated manometers of the classes that embrace the entire range of pressures used). However, the most appropriate solution seems to be a gauge that is based on the pressure-compensation method. In that case several advantages can be gained: 1) almost no extra amount of the D-T mixture will be involved; 2) the space in which the pressure is measured is separated from the working volume of the chamber. The respective evaluation of the basic characteristics of the pressure pickup is reasonable to perform only after the final choice is made. 
3.2. Additional Helium Flow Cryostats. As crude estimates show for the technological sequence accepted (see Sec. 5), the $20 \mathrm{~K}$ sink would be insufficient to ensure the fast cooling needed during cryopumping of the mixture from the chamber to the auxiliary capacity. We suggest two independent flow refrigerators with a single communication system (see Figs. 1 and 2). However, a more intricate scheme can be suggested with only one flow refrigerator placed at the syringe unit. The heat removal from the chamber unit will be performed via a heat link of controllable thermal conductance. The design in that case will be more involved and require separate detailed calculations.

\section{P-V-T Relationships: Thermodynamic Calculations}

Our primary thermodynamic calculations reported previously [1] have been performed for pure $n-D_{2}$. Here we report corrections to those results for the equimolar D-T mixture. Our explanations will be illustrated by Fig. 4, where we plot the known experimental data for the saturated pressure as a function of temperature for pure deuterium (solid squares) [2] and pure tritium (empty squares) [3] from $15 \mathrm{~K}$ up to the critical points. Our calculations regard the D-T mixture of the particular density of $\bar{\rho}=13600 \mathrm{~mole} / \mathrm{m}^{3}$.

D-T mixtures are fairly close to ideal, that is the total pressure in the mix is a sum of the partial pressures whose values are independent of the presence of the other gas(es). This problem was addressed by Souers [4]. If we use his formulas to estimate the crossterm virial coefficient as well as the fluid binary-mixing parameters, then for $\bar{\rho}$ we obtain that the relative value of the correction to the pressure for $T>35 \mathrm{~K}$ will be below $1 \%$. However, there are some doubts as to the reliability of Souers' estimates for our particular case. First, those estimates obtained for $T \leq 30 \mathrm{~K}$ might be misleading for the phase areas near the critical point: see how very close to the critical points of both components the thermodynamic path (the solid curve in Fig. 4) of the mixture of interest to us approaches. Second, even for the interval quoted above, Souers' estimates conflict seriously with experimental, however uncertain, findings [5,6]; the pressure corrections estimated on the basis of those experimental data turn out to be 2-3 times larger than Souers'. But anyway, the magnitude of the corrections is luckily rather small to the extent that for our purposes we may rely on the evaluations for $D_{2}$, which can be performed to a very good accuracy. To this end we employed the multi-parameter representation of the deuterium P-V-T data by Prydz et al. [7], which exhibit very good applicability even for areas close to the critical point. At higher temperatures we used less involved representations, mostly the virial one with the coefficients borrowed from Souers [4].

Thus, we calculated the thermodynamic path (an isopyc for $\bar{p}=13600$ mole per $\mathrm{m}^{3}$ ) for pure normal deuterium (an exemplary point is shown in Fig. 4 as the solid circle) and then shifted the path (solid curve) upward by $1.5 \%$. When we cool down the mix, the phase point of our system moves along this path to meet the saturated vapor-liquid equilibrium line. If the system were pure deuterium, it would have met the pure- $D_{2}$ line at the point 
shown in Fig.4 as the smaller empty circle and then moved along the equilibrium line, the system having become inhomogeneous (vapor + liquid) upon condensation. For the equimolar D-T mixture, the phase path hits the equilibrium region (now it is not a line but a strip, not very wide because of the closeness of the properties of the components) at the point shown as the larger empty circle. Then the phase point will follow a more intricate path involving partial fractionation. The particular finer details of the path shape depend on the cooling procedure.

The maximum amount of tritium allowed by the regulations to be handled without special protection is $1000 \mathrm{Ci}$ at normal conditions, or (recalling that the density $\rho_{0}$ of the ideal gas at those conditions is $44.6 \mathrm{~mole} / \mathrm{m}^{3}$ ) 0.731 mole. Let us compare this figure with what follows from our calculations. The total amount of the D-T mixture involved in a single fill cycle constitutes:

- for the syringe design without pressure gauge, $4.1 \times 10^{-3}$ moles;

- for the constant-volume capacity design without pressure gauge, $5.6 \times 10^{-3}$ moles;

- for the constant-volume design with pressure gauge, $(8-11) \times 10^{-3}$ moles.

The margin is thus very good.

\section{Process Flow Chart}

The idea behind the design, suggested here along the guidelines specified in Scope of Work of the Agreement, pivots on feasibility of situations when the two working capacities are maintained for a long enough time at a very high temperature difference. Moreover, at certain moments the technological requirements demand that the temperature be varied rather fast. All these factors necessitate use of a combined cooling technique. Namely, in addition to the standard heat sink bus, thermally linked to a liquid He bath, we suggest a liquid-helium flow refrigerator, whose cooling tubing is wound over (or worked out in) the body of both units.

In this Section we formulate a detailed process sequence as we find proper (see Fig. 5). The time axis does not correspond to any real scale. The time variations of the temperature in the chamber, $T_{\text {cham }}$, the temperature in the syringe, $T_{y y r}$, and the pressure $P$, which is essentially the same in both capacities, are schematic.

Stage I. We consider the situation when the assembly is working in cycles. At the beginning of this initial stage the chamber at room temperature already houses a new empty capsule. The temperature inside the syringe is around $20 \mathrm{~K}$. The pressure is that of saturated vapor (about $0.3 \mathrm{bar}$ ). The syringe is partly filled with liquid. At this stage, intake from a membrane pump occurs. The amount of the working substance is restored. (Note: Since the amount of substance to be restored is rather small, several cycles can be performed without syringe refill; the final pressure needed at $300 \mathrm{~K}$ to fill the capsule can be attained by a slight overheating of the syringe and/or chamber as compared to the previous cycle.) 
Stage II. The temperature of the chamber is kept at $300 \mathrm{~K}$. The temperature of the syringe is raised in controlled manner so as to keep the pressure drop across the capsule wall below 3-5 bar. The pressure in the system rises till the necessary amount penetrates into the capsule. Note that during stage II the temperature in the chamber is always 300 $\mathrm{K}$, which ensures maximum permeability of the capsule wall.

Stage III. As soon as the capsule contains the necessary amount (or a bit sooner) the temperature in both capacities is decreased rather abruptly to about 150-180 $\mathrm{K}$ (this temperature level depends on penetration mechanism, or on the penetration activation energy if the mechanism is diffusive). Such a decrease virtually stops permeation but leaves the substance still in an easily volatile state.

Stage IV. This stage is needed to evacuate the chamber in order that the filled capsule can be removed. The pressure at the beginning of Stage IV is slightly above 200 bar. Now the temperature of the syringe is varied from $150 \mathrm{~K}$ down to $20 \mathrm{~K}$, whereas the temperature of the chamber is decreased at a slower rate down to $55 \mathrm{~K}$. Virtually all the substance is cryopumped back to the syringe. At the end of Stage IV the capsule is removed and an empty one is placed instead.

Stage V. Now the chamber is warmed up to room temperature, maintaining the syringe at low temperature. The cycle is completed.

\section{Conclusions}

1. The low-inventory design is feasible. Quite realistic evaluations allow us to expect that the total amount of fuel used in one cycle (or even several cycles) will never exceed $3 \times 10^{-2}$ moles.

2. There are several problems to be solved before detailed engineering design is to be started. Such problems concern:

- rate of permeation, or in other words, the timwe needed to fill a capsule, which is a very important factor that could influence the engineering design of the setup;

- details of the capsule removal procedure, which will also be decisive for specific choices in engineering design and calculations;

- although the process of varying the temperatures during stages II and IV can be controlled with a computer-aided facility, developed using calculated and experimental data, it seems that a more direct method of monitoring of the running values of the pressure (or the mass) in the capsule during permeation is of a primary importance.

3. The objectives of the task necessitate use of a combined refrigeration (stationary cooling buses + flow refrigerator) of both the fill chamber and the so called syringe block. 


\section{References}

1. 1st Quarterly Report, Agreement B 336593 (1997).

2. A. S. Friedman, D. White, and H. L. Johnston, J. Amer. Chem. Soc. 73, 1310 (1951).

3. E. R. Grilly, J. Amer. Chem. Soc. 73, 843 (1951).

4. P. C. Souers, Cryogenic Hydrogen Data Pertinent to Magnetic Fusion Energy, LLNL Rep. UCRL-52628 (1979).

5. R. H. Sherman, J. R. Bartlit, and R. A. Briesmeaister, Cryogenics 16, 611 (1976).

6. P. C. Souers, E. M. Kelly, and R. T. Tsugawa, Trans. Amer. Nucl. Soc. 28, 202 (1978).

7. R. Prydz, R. D. Timmerhaus, and R. B. Stuart, Adv. Cryogen. Eng. 13, 384 (1968). 


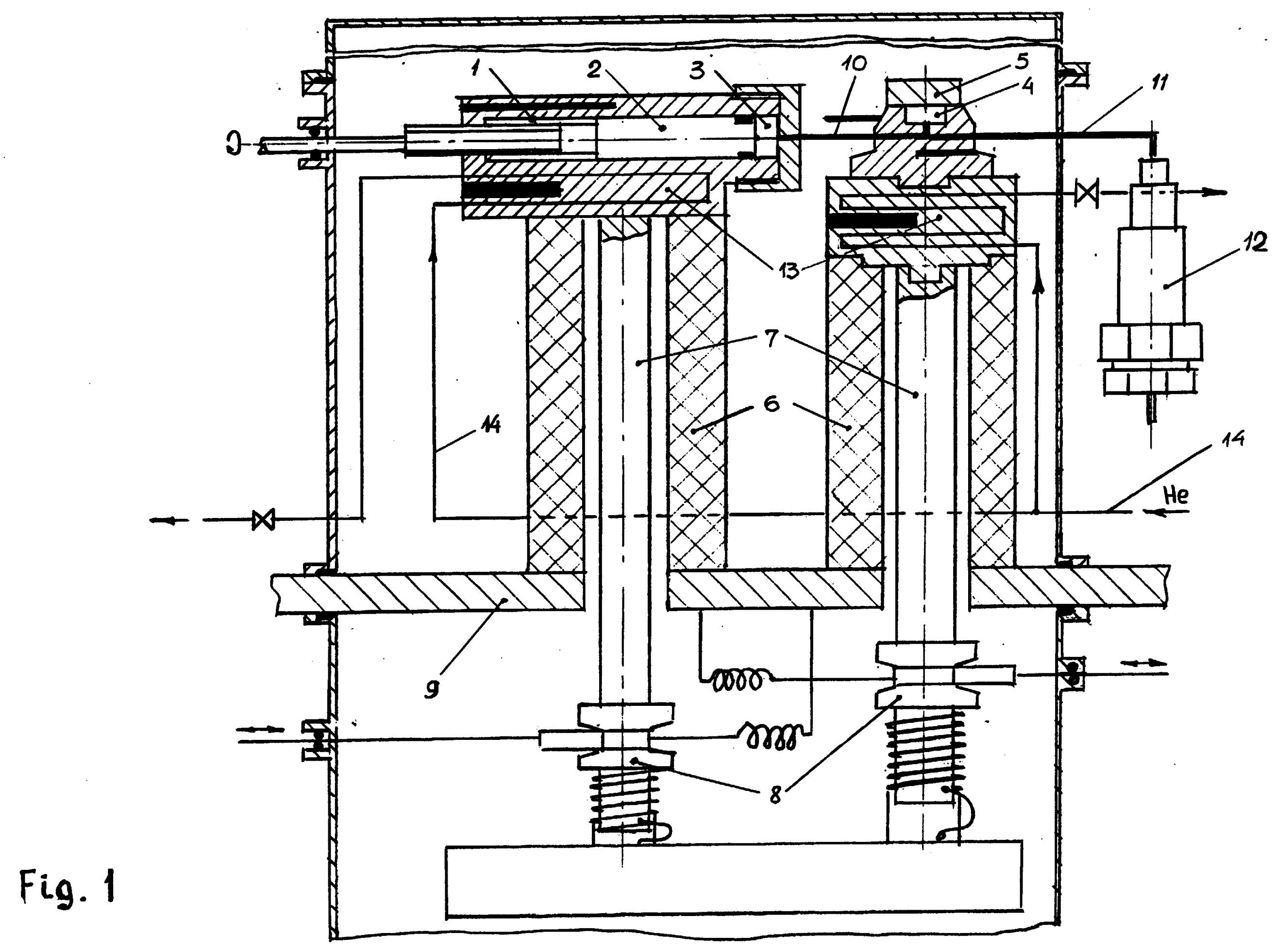




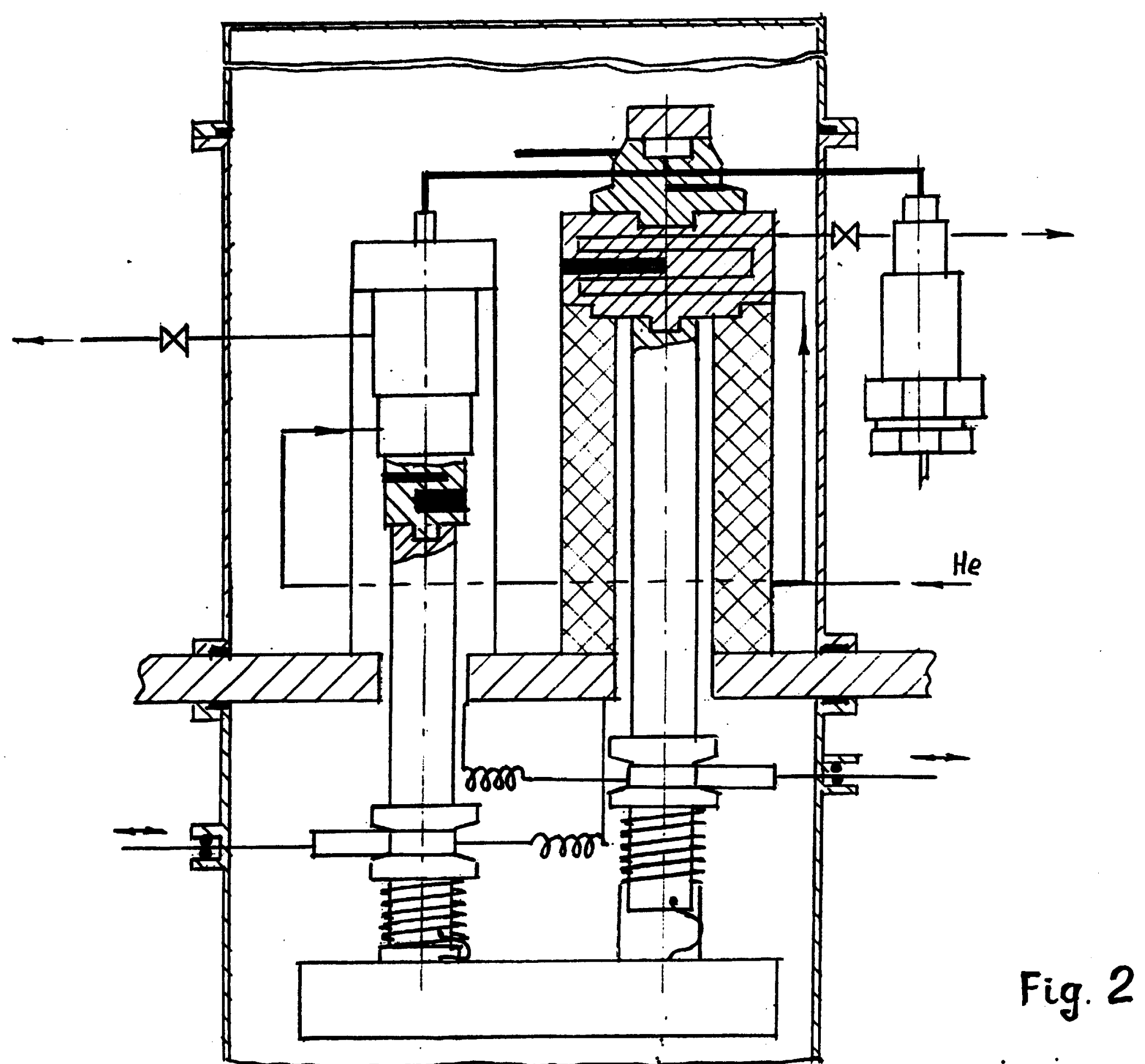



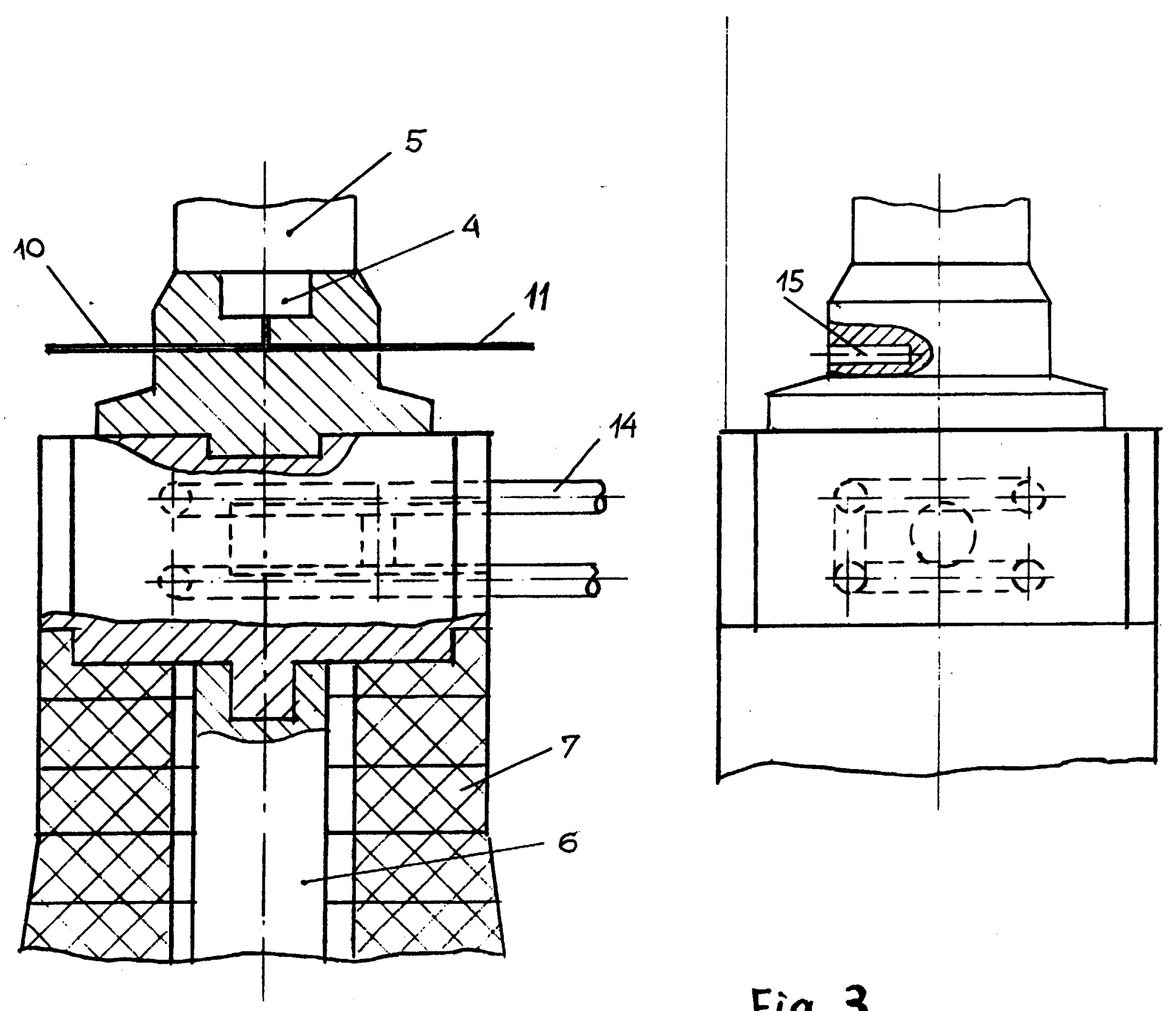

Fig. 3 


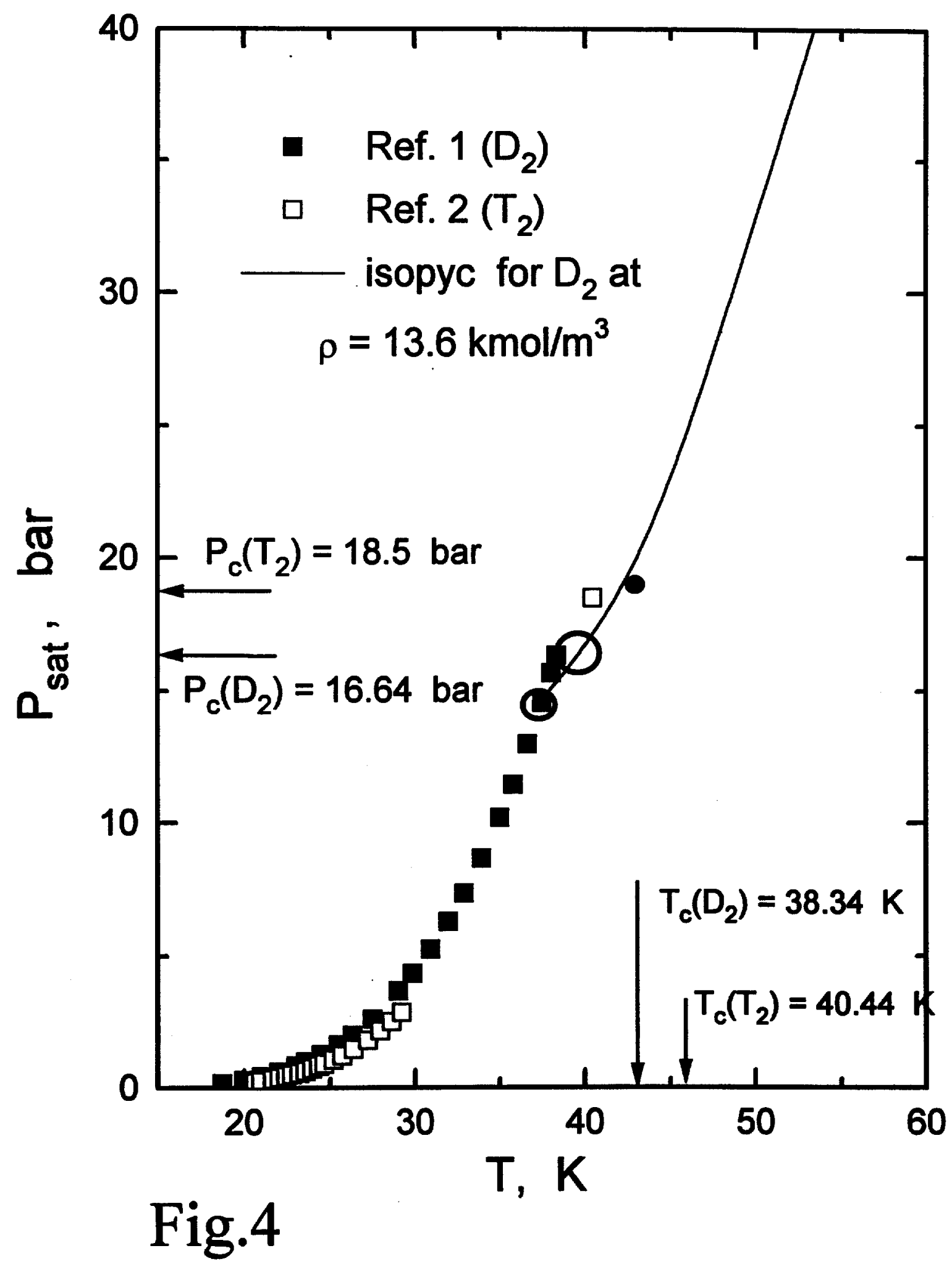




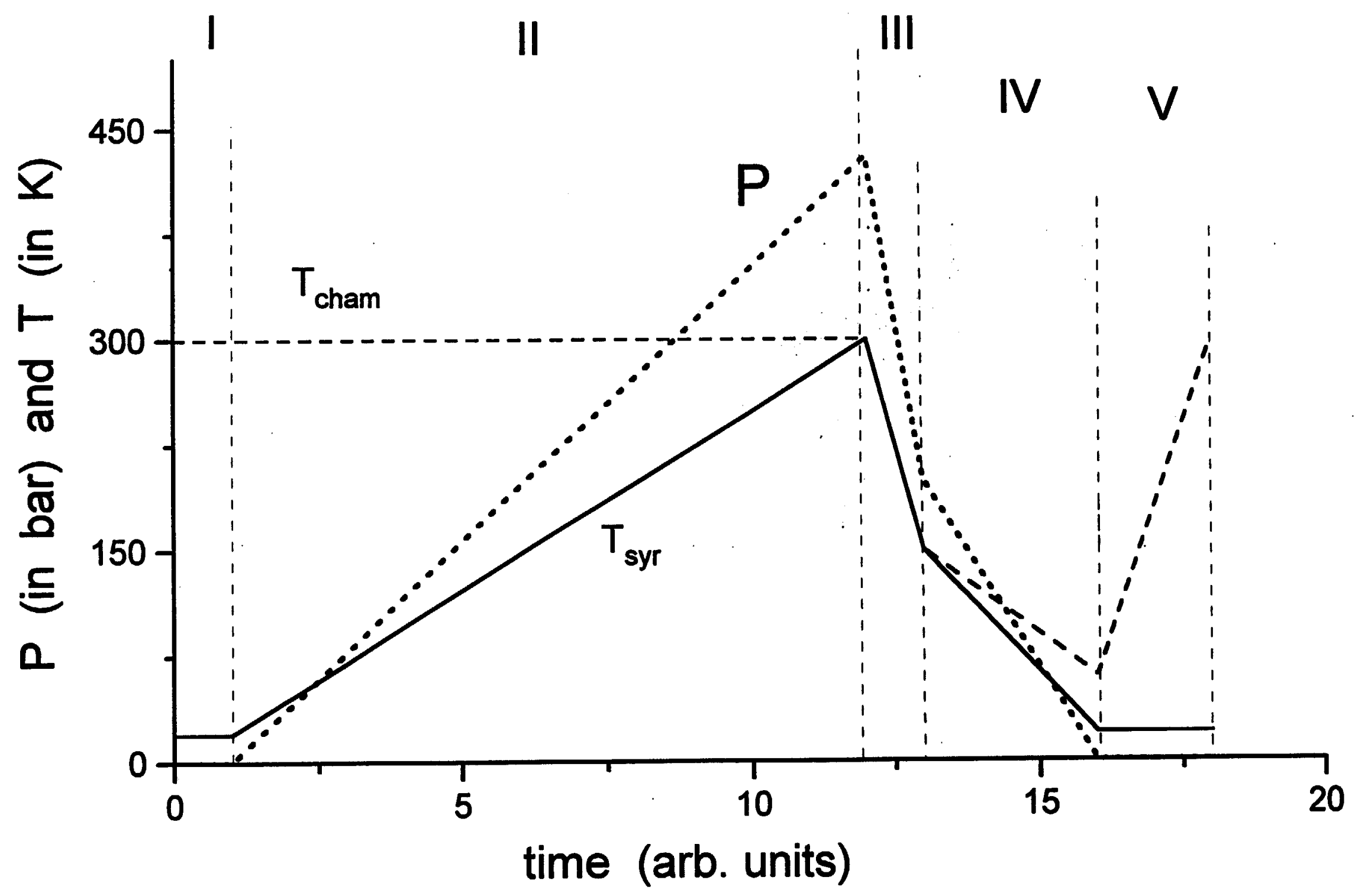




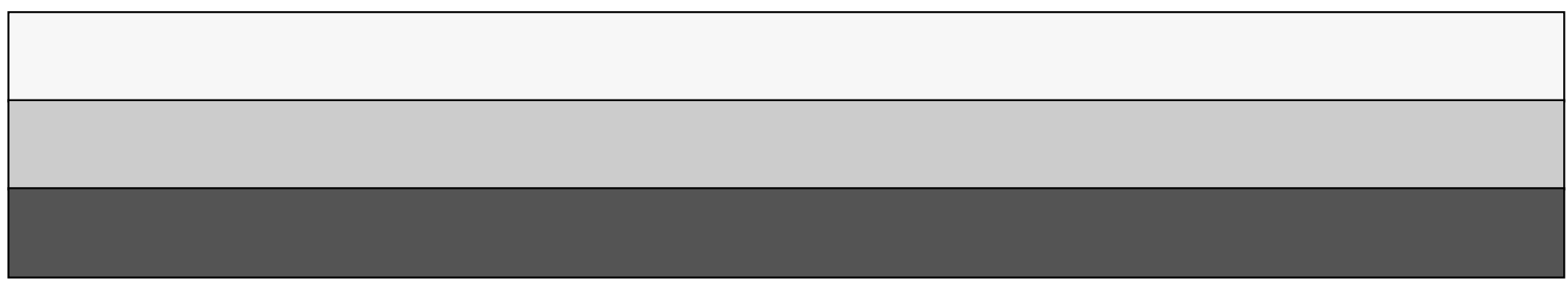

